\title{
LOW-EMISSION BERTH ALLOCATION BY OPTIMIZING SAILING SPEED AND MOORING TIME
}

\author{
Zhi-Hua HU \\ Shanghai Maritime University, China \\ Received 26 May 2016; revised 23 September 2016, 28 February 2017; accepted 5 April 2017

\begin{abstract}
To investigate the relations among delay times (weighted by vessels' handling times), the emissions during the vessels' sailing and mooring in a Berth Allocation Problem (BAP) where the berth times and sailing speeds are formulated as decision variables. The vessels' delay times are computed comparing to the vessels' Expected Departure Times (EDTs); the sailing emission is determined by the sailing speed and distance; the mooring emission is positive to the mooring time at terminal. Multi-objective mixed-integer programs are established, and the nonlinear functions between emissions and sailing speeds are transferred to linear ones by the Second-Order Cone Programming (SOCP) method. Solution methods are further developed based on $\varepsilon$-constraint and stage-based methods by considering the preferences of objectives. Four groups of experiments are conducted to demonstrate the formulations, effects of vessels' handling times and EDTs on the solutions, and the reduced emissions affected by the number of vessels in the schedules. Experimental results demonstrated that the efficiency purpose is not absolutely conflict with the environment purposes for some instances, and so they can be pursued at the same time; improving the vessels' handling efficiency help expand the ranges of berth times and sailing speeds, resulting in reducing the delay times and emissions; advancing the EDTs can improve the terminal operators' service quality to shipping companies, while the weighted delay times and emission may be increased.
\end{abstract}

Keywords: container terminal, berth allocation problem, shipping, fuel consumption, low-emission logistics, logistics management.

\section{Introduction}

The data for the world showed that ocean-going vessels accounted for $27 \%$ of $\mathrm{CO}_{2}, 98.9 \%$ of $\mathrm{SO}_{\mathrm{x}}, 48 \%$ of $\mathrm{NO}_{\mathrm{x}}$ and $58.3 \%$ of fine particles of all port emissions at 2010 (Starcrest Consulting Group 2011). Therefore, many terminal operators, shipping companies and environmentrelated authorities are very concerned with reducing emissions and pollution. To reduce operational costs, shipping companies also strive to reduce the vessel fuel consumption. The economic (e.g., fuel consumption) and environmental concerns (e.g., vessel emissions) influence the decisions performed markedly by the terminal operators and shipping companies. Dedes et al. (2012), Song and Xu (2012), Fagerholt et al. (2010) developed some decisionmaking strategies for port environment protection and optimization of vessel fuel consumption. Terminal operators and shipping companies are interacted by berth allocation schedules, which allocate the terminal's operational resources to vessels' loading and unloading operations.
The Berth Allocation Problem (BAP) optimizes the terminal's operational cost and service quality generally by considering the acceptance of shipping companies. Usually, the Expected Arrival Times (EATs) and Expected Departure Times (EDTs) are given by fixed time windows, which are set to ranges of berth times and finishing times of handling the vessels by the terminal. On these premises, the sailing speeds (or, sailing times for given sailing distances) and mooring times at terminals are determined in the vessels' schedules by the shipping companies. However, these two factors directly determine the emissions during the vessels' sailing and mooring periods. The berth times are medium variables that coordinate these two factors. Therefore, within the accepted arrival and departure time windows, the terminal operators can further suggest optimal berth times to the shipping companies for adjusting the vessels' sailing speeds that also affect the mooring times between the arrival and berth times. In other

*E-mail: zhhu@shmtu.edu.cn

Copyright $\odot 2020$ The Author(s). Published by Vilnius Gediminas Technical University

This is an Open Access article distributed under the terms of the Creative Commons Attribution License (http://creativecommons.org/licenses/by/4.0/), which permits unrestricted use, distribution, and reproduction in any medium, provided the original author and source are credited. 
words, the possible reduction of emissions is achieved by the coordination of terminal operators and shipping companies. Under this premise, three objectives are identified and formulated in the multi-objective programs, namely, delay times weighted by vessels' handling times, emissions in the sailing periods and emissions in the mooring times.

The remainder of this paper is organized as follows. Section 1 provides a review of related studies on BAPs and low-emission maritime logistics. Section 2 formally describes the procedures for calculating fuel consumption and emissions from vessels. Section 3 presents the proposed mathematical formulations. Section 4 estimates the related parameters and data for experiments in Section 5. This work concludes with last section and future research directions are suggested.

\section{Related studies}

Related to this work, two research streams are involved: BAP and low-emission maritime logistics.

\subsection{BAP}

For comprehensive overviews on container terminal operations and maritime logistics, see the reviews given by Vis and De Koster (2003); Steenken et al. (2004); Stahlbock and Voß (2008); Fransoo and Lee (2013). The BAP is very important for ports' operations management and is also the basis for making other plans on container scheduling decisions by shipping liners (Choi et al. 2012). The BAP generally refers to the assignment of quay space and service time to vessels that have to be unloaded and loaded at a terminal in seaside operations. The seaside operations in container terminals comprise the BAP, the quay crane assignment problem, and the quay crane scheduling problem. They determine the vessels' stay times at container terminals, which basically reflect the service quality promised to shipping companies and thus the competitiveness of a terminal. In BAPs, a berth layout is given together with a set of vessels that have to be served within a planning horizon. The vessels must be moored within the boundaries of the quay and two vessels cannot occupy the same quay space at a time. In the basic optimization problem, berthing positions and berthing times have to be assigned to all vessels. A variety of optimization models for berth allocation have been proposed to capture realworld features of practical problems. Bierwirth and Meisel (2010), and further Bierwirth and Meisel (2015) proposed a scheme for classifying such models according to four attributes, namely spatial, temporal and handling time attributes, and the performance measure. In this scheme, the proposed model in this study formulated the BAP with continuous layout, dynamic arrivals, and fixed handling times of vessels.

The continuous dynamic BAP with fixed handling times has been investigated in a number of studies. Guan and Cheung (2004) developed a tree search procedure to minimize the total weighted port stay time of vessels.
Wang and Lim (2007) minimized the penalty cost for rejected vessels and the optimal berthing positions are also pursued. A stochastic beam search algorithm is presented that can solve instances with up to 400 vessels. A further objective, namely the minimization of tardiness of vessels was treated by Park and Kim (2003), and Kim and Moon (2003). Several solution methods were proposed for this problem, including a sub-gradient method (Park, Kim 2002) and a simulated annealing approach (Kim, Moon 2003). Lim (1998) formulated a problem, where the berthing times of vessels are already set by the arrival times. Instead, the suitable berthing positions are to be determined and the goal is to minimize the maximal quay length required to serve vessels in accordance with the schedule.

As to the solution methodology for the continuous BAP, Kim and Moon (2003) proposed a simulated annealing method. Park and Kim (2002) employed a sub-gradient optimization method. Guan and Cheung (2004) developed a heuristics algorithm to minimize the total weighted completion time. Wang and Lim (2007) developed a beam search method for solving BAP. Recently, Bierwirth and Meisel (2015) provided an overview of the methods that are used for solving the BAP models. The heuristic approaches dominate the solution methods of BAPs because the BAPs are known to be Non-deterministic Polynomialtime hardness (NP-hard) as asserted by Lim (1998). Exact methods were applied in only one fourth of the approaches, ranging from mixed-integer linear programs combined with standard solvers to sophisticated branching-based algorithms. Among the heuristic approaches, genetic algorithms and evolutionary algorithms take over about forty percent, e.g., Rodriguez-Molins et al. (2014); Chang et al. (2010); Seyedalizadeh Ganji et al. (2010); Yang et al. (2012). The rest methods comprise other meta-heuristics like Tabu search and simulated annealing as well as the heuristics like local search techniques and greedy rules, e.g., Zhen et al. (2011); Zeng et al. (2011a, 2011b) and Elwany et al. (2013). However, the above methods can not guarantee the optimality of solutions. So solution methods based on standard mixed-integer program solvers do also prevail, e.g., Raa et al. (2011); Hendriks et al. (2010).

\subsection{Low-emission BAP and maritime logistics}

As reviewed by Bierwirth and Meisel (2015), to control the fuel consumption of a vessel on its way to a terminal, Golias et al. (2009) and Du et al. (2011) considered the arrival times as decision variables in the BAP. There, the arrival time can be varied within a certain range, in correspondence to the sailing speed of the vessel and the fuel consumption. The approach can be used for trading off the fuel consumed for going to and waiting at a terminal under the objective of meeting a given due date. Du et al. (2011) proposed an emission estimation model that converts fuel consumption into GreenHouse Gas (GHG) emissions. Experiments with artificial test instances indicate that substantial savings can be gained from such an integration. To achieve a better trade-offs of fuel con- 
sumption, emissions, and service times, Hu et al. (2014) combined berth allocation with quay crane assignment. Again, arrival times are considered as decision variables used to determine the optimal sailing speeds. Next to this, the assignment of quay cranes to vessels is used for adjusting the handling times, which in turn impact the arrival times needed for ensuring timely departures of the vessels from the terminal.

As described above, the low-emission BAP connects with the sailing periods and the shipping liner scheduling problems. Usually, related to BAPs, three low-emission scenarios in operations research can be identified: sailing, mooring and vessel handling at container terminals. The vessels' handling emissions are related to equipment assignment and usage; the mooring emission relates to the mooring time at terminals generally; and the sailing emission is determined by the sailing speeds primarily. In equipment selection, engines and fuel usage are important to emission and cost control. However, mooring time has to include typical berthing and deberthing delays. The distances from mooring/anchorage area to berth line could be short, medium and large depending on the port configuration. This operation can be mainly performed by port tugs or by means of own vessel's engine(s).

In the shipping industry, speed reduction has become a very popular operational measure to reduce fuel consumption and can obviously be used to curb emissions. Kontovas and Psaraftis (2011) examined this operational scenario. Since the time at sea increases with slow steaming, there is a parallel and strong interest to investigate possible ways to decrease the stay time in a terminal. One way to do so is to reduce the service time at a terminal. Another possible way to minimize disruption and maximize efficiency is the prompt berthing of vessels upon arrival. A related berthing policy is investigated as a measure to reduce the standing times of vessels at the terminals. The objective of reducing emissions along the maritime inter-modal container chain is investigated in operational costs and other service attributes. Gibbs et al. (2014) investigated the actions of reducing the GHG emissions associated with the end-to-end maritime transport chain based on both the analysis of secondary data and information. The analyses indicated that emissions generated by vessels during their voyages between terminals are of a far greater magnitude than those generated by the terminal activities. Thus, while reducing the terminals' own emissions is worthwhile, the results suggested that terminals might have more impacts through focusing their efforts on reducing sailing emissions.

Given the prospect of an increase in $\mathrm{CO}_{2}$ emissions caused by sailing, the debate surrounding regulations relating to emissions in this sector is intensifying in several international fora. The International Maritime Organization (IMO) and the European Union are discussing the introduction of a market-based measure for maritime transport. Franc and Sutto (2014) focused on the principle of a cap-and-trade system and explored the potential impacts of the implementation of such a measure on the organization of containerized shipping lines and European ports. The results demonstrated significant and differentiated effects between various scenarios.

Strict limits on the maximum sulphur content in fuel used by vessels have been imposed recently in some Emission Control Areas (ECAs). In order to comply with these regulations many vessel operators will switch to more expensive low-Sulphur fuel when sailing inside ECAs. Since they are concerned about minimizing their costs, it is likely that speed and routing decisions will change because of this. Fagerholt et al. (2015) developed an optimization model to be applied by vessel operators for determining sailing paths and speeds that minimize operating costs for a vessel along a given sequence of terminals. On some shipping routes, this might give a considerable increase of the total amount of fuel consumed and the $\mathrm{CO}_{2}$ emissions. Lindstad et al. (2015) assessed cost as a function of abatement options in maritime ECAs. This study indicates that there is no single answer to what is the best abatement option. The best option will be a function of engine size, annual fuel consumption in the ECAs and the foreseen future fuel prices.

Data-driven analysis is increasingly focused on in emission assessment and optimization. Schrooten et al. (2009) presented the methodology for a comprehensive maritime transport database of activity data, specific energy consumption, emission factors and total emissions. The model is built upon three modules: the fleet, transport activity and the emission modules. The emission module calculates the energy consumption by using the $\mathrm{CO}_{2}, \mathrm{NO}_{\mathrm{X}}, \mathrm{SO}_{2}, \mathrm{CO}, \mathrm{HC}, \mathrm{CH}_{4}, \mathrm{NMHC}, \mathrm{PM}$ emissions from the resulting maritime activities. Yau et al. (2012) determined minute-by-minute high-resolution speed profiles of container vessels on four major routes using the automatic identification system (AIS). The activity-based vessel emissions of $\mathrm{NO}_{\mathrm{x}}, \mathrm{CO}, \mathrm{HC}, \mathrm{CO}_{2}, \mathrm{SO}_{2}$ and PM10 were estimated using derived vessel speed profiles, and the results were compared with those using the speed limits in ECAs.

Close to $70 \%$ of maritime emissions are emitted within $400 \mathrm{~km}$ of land, consequently imposing problems with air quality in coastal areas and ports handling high volumes (Svindland 2018). However, in this study, mainly the short sea transport is considered.

Comparing with the state-of-the-art literature, this paper makes a comprehensive study on the low-emission BAP. The weighted delay times, sailing emission and mooring emission were directly formulated as three objectives in the proposed models.

\section{Fuel consumption and emissions}

A schedule returned by solving a BAP is closely related to a shipping schedule. Arrival times of vessels determined by a shipping schedule influence the berthing plan markedly; inversely, the berthing plan may delay the departure of a vessel, which influences the shipping schedule significantly. Additionally, the quay crane allocation schedule 
determines the vessels' operational times, affecting at-port duration and departure time directly. These further affect the amount of emissions from vessels and the shipping schedule. Therefore, a close relationship between the BAP and shipping schedules requires that the terminal operators and shipping companies work together. A terminal operator may ask a vessel to slow or accelerate to meet a specific loading/unloading time slot to minimize fuel consumption and emissions while sailing and/or moored. Notably, terminal operators should consider operational efficiency for the vessels that they serve, improve the utilization degree of terminal-related resources, and negotiate with shipping companies to optimize the arrival time for vessels.

Figure 1 presents the times given in an example of BAP for describing the delay and emissions. The vessels are berthed in a bi-dimensional time-space diagram. The schedule is generated at present (time is zero). The minimal EAT falls in the range limited by the "Min EAT" and the "Max EAT", which are determined by the sailing speed limits of the vessel. If the vessel arrives at the time "Arrival time", then the "Sailing time" can be determined, which induces the sailing emissions. After the vessel arrives and before it berths at a specific berthing position, mooring emission is produced. After the vessel is handled by the terminal, it departures from the terminal. This departure may be delayed comparing to the vessel's EDT.

As described above, the sailing time is determined by the sailing speed when the sailing distance is known. Then, the fuel consumption of vessels when sailing is affected mainly by the distance to the terminal and sailing speed (Du et al. 2011; and $\mathrm{Hu}$ et al. 2014). Further, the sailing distance and sailing speed determine the arrival time at port directly.

A vessel set is denoted by $V$ and indexed by $i, j$. A berthing plan begins at time zero, and arrival time of vessel $i$ is denoted by $a_{i}$; for a vessel $i$, its shipping company controls the arrival time $a_{i}$ in an interval $\left[\underline{A}_{i}, \bar{A}_{i}\right]$ by adjusting the sailing speed, where: $\underline{A}_{i}$ and $\bar{A}_{i}$ are determined by its maximum sailing speed and minimum sailing speed individually; the distance from vessel $i$ to a terminal is denoted by $M_{i}$, when the berthing plan begins, where $i \in\{1,2, \ldots, N\}$ denotes a vessel set. Fuel consumption of vessel $i$ then can be derived by Equation (1), where: $\lambda_{i}^{0}$ and $\lambda_{i}^{1}$ are positive regression coefficients, and $u_{i} \in\{3.5,4,4.5\}$. The original form of Equation (1) was derived by $\mathrm{Du}$ et al. (2011). In addition, later the formula was then used by $\mathrm{Hu}$ et al. (2014). For feeders $-u_{i}=3.5$; for medium-sized vessels $-u_{i}=4$; for jumbo vessels $-u_{i}=$ 4.5. To present the vessels by their sizes, three sets are denoted as $V^{\text {Feeder }}, V^{\text {Medium }}$ and $V^{\text {Jumbo }}$.

There is an optimal sailing speed $\tilde{s}$ when the fuel consumption is minimized, which can be achieved by minimizing fuel consumption $f_{i}$ in Equation (1). A vessel can decelerate to save fuel consumption when the speed exceeds $\tilde{s}$, or it can accelerate to save fuel when the speed is slower than $\tilde{s}$. The optimal sailing speed $\tilde{s}_{i}$ of vessel $i$ can be derived by Equation (2). Figure 2 presents two curves for the relations between the fuel consumption and sailing speed.

$$
\begin{aligned}
& f_{i}=\left(\lambda_{i}^{0}+\lambda_{i}^{1} \cdot\left(\frac{M_{i}}{a_{i}}\right)^{u_{i}}\right) \cdot a_{i}=\left(\lambda_{i}^{0}+\lambda_{i}^{1} \cdot\left(s_{i}\right)^{u_{i}}\right) \cdot \frac{M_{i}}{s_{i}}= \\
& \lambda_{i}^{0} \cdot a_{i}+\lambda_{i}^{1} \cdot M_{i}^{u_{i}} \cdot a_{i}^{1-u_{i}} \\
& \tilde{s}_{i}=\left(\frac{\lambda_{i}^{0}}{\lambda_{i}^{1} \cdot\left(u_{i}-1\right)}\right)^{\frac{1}{u_{i}}} \cdot
\end{aligned}
$$

Emissions from vessels mainly consist of emissions while moored and sailing. Due to a lack of relevant data, calculating emissions precisely is difficult. According to Du et al. (2011), emissions of vessel $i$ while sailing $e_{i}^{\text {Sail }}$ can be calculated by Equation (3), where: $f_{i}[\mathrm{~kg}]$ is fuel consumption of vessel $i$ while sailing; $E_{\text {in }}^{\text {Sail }}$ [g/kg fuel] is an emission factor of the $n$-th pollutant for vessel $i$. This study mainly considers emissions of $\mathrm{CO}_{2}, \mathrm{NO}_{\mathrm{x}}$ and $\mathrm{SO}_{\mathrm{x}}$.

$$
\begin{aligned}
& e_{i}^{\text {Sail }}=\sum_{n}\left(f_{i} \cdot E_{\text {in }}^{\text {Sail }}\right)=f_{i} \cdot \sum_{n}\left(E_{n}^{\text {Sail }}\right) ; \\
& E^{n \text { Sail }}=\sum_{n}\left(E_{n}^{\text {Sail }}\right) ; \\
& e_{i}^{\text {Sail }}=f_{i} \cdot E^{n \text { Sail }} .
\end{aligned}
$$

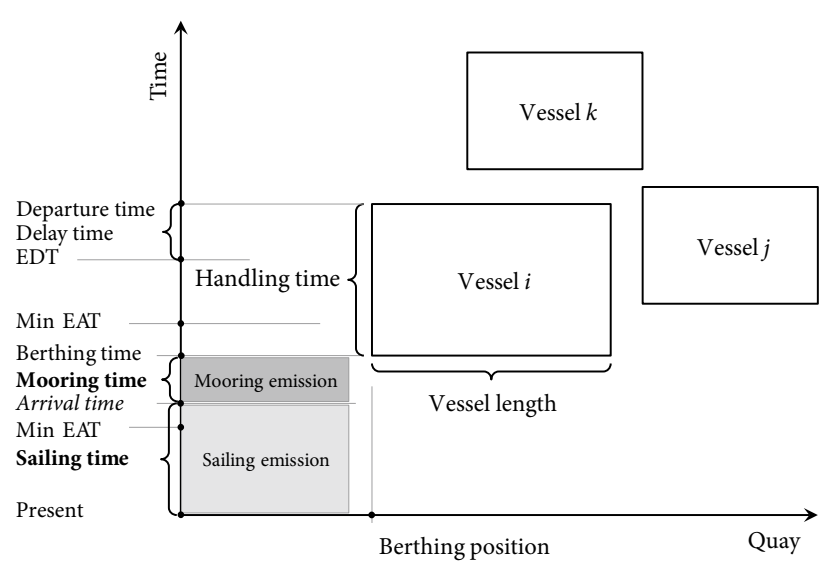

Figure 1. The times describing the delay and emissions in a BAP

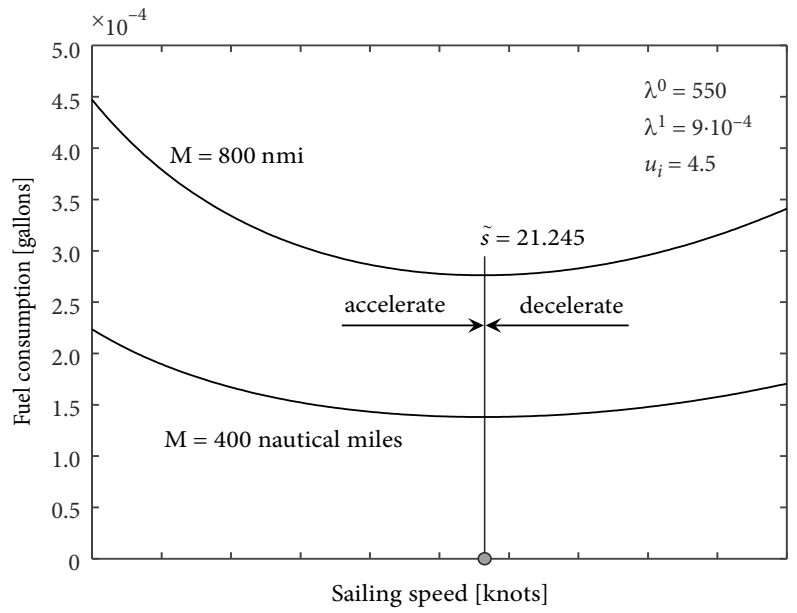

Figure 2. Fuel consumption versus sailing speed 
The standing time at port is the duration between operations start time and a vessel's depart time. Operating cost (to keep relevant equipment operating, a vessel consumes energy and discharges emissions when waiting at port and being served) and opportunity cost (profits gained by utilization of the standing time) are also influenced by the standing time at the terminals. Golias et al. (2009) and Du et al. (2011) used standing times at the terminals to measure emissions from moored vessels indirectly. In this work, by using the data from Starcrest Consulting Group (2011), emissions while moored can be calculated directly based on wait time for berthing. The emissions of vessel $i$ while moored $e_{i}^{\text {Moor }}$, can be calculated by Equation (4), where: $E_{i}^{P O}[\mathrm{~kW} / \mathrm{hp}]$ represents the rated power of the engine of vessel $i ; A C_{i}[\mathrm{~h}]$ is the average activity time of each engine of vessel $i ; E^{L F}$ is a load ratio (ratio of average power used during normal operations to maximum rated power); $E_{\text {in }}^{\text {Moor }}[\mathrm{g} / \mathrm{kW} \cdot \mathrm{h}]$ is an emission factor of the $n$-th pollutant for vessel $i$ while mooring; $E_{n}^{\text {Fuel }}$ is a fuel correction factor of the $n$-th emission factor to reflect changes in fuel properties over time; $E_{i}^{\text {Engine }}$ is the number of engines of vessel $i$. Similarly, this work mainly considers emissions of $\mathrm{CO}_{2}, \mathrm{NO}_{\mathrm{x}}$ and $\mathrm{SO}_{\mathrm{x}}$ (see the reference value of related factors of emissions - Starcrest Consulting Group 2011).

$$
\begin{aligned}
& e_{i}^{\text {Moor }}=\sum_{n}\left(E_{i}^{\text {PO }} \cdot t_{i}^{\text {active }} \cdot E^{L F} \cdot E_{\text {in }}^{\text {Moor }} \cdot E_{n}^{\text {Fuel }} \cdot E_{i}^{\text {Engine }}\right)= \\
& t_{i}^{\text {active }} \cdot\left(E_{i}^{\text {PO }} \cdot E^{L F} \cdot E_{i}^{\text {Engine }} \cdot \sum_{n}\left(E_{\text {in }}^{\text {Moor }} \cdot E_{n}^{\text {Fuel }}\right)\right)= \\
& t_{i}^{\text {active }} \cdot\left(E_{i}^{\text {PO }} \cdot E^{L F} \cdot E_{i}^{\text {Engine }} \cdot \sum_{n}\left(E_{n}^{\text {Moor }} \cdot E_{n}^{\text {Fuel }}\right)\right) ; \\
& E^{n \text { Moor }}=\sum_{n}\left(E_{n}^{\text {Moor }} \cdot E_{n}^{\text {Fuel }}\right) ; \\
& E_{i}^{\text {Moor }}=\left(E_{i}^{\text {PO }} \cdot E_{i}^{\text {Engine }}\right) \cdot\left(E^{L F} \cdot E^{\text {Moor }}\right) ; \\
& e_{i}^{\text {Moor }}=t_{i}^{\text {active }} \cdot E_{i}^{\text {Moor }} .
\end{aligned}
$$

\section{Mathematical formulations}

\subsection{Second-order cone programming transformation}

Consider a function $f=a^{1-u}$ in a minimization objective (Equation (1)). It can be represented by $f=q$ subject to $a^{1-u_{i}} \leq q$. The constraint is transferred to $1 \leq q \cdot a^{u-1}$. When $u \in\{3.5,4,4.5\}$, the constraint is then transferred to $1 \leq q \cdot a^{2.5}, 1 \leq q \cdot a^{3}$ and $1 \leq q \cdot a^{3.5}$ individually. To remove the fraction exponents, they are transferred to $1 \leq q^{2} \cdot a^{5}$, $1 \leq q \cdot a^{3}$ and $1 \leq q^{2} \cdot a^{7}$ individually. In the following, the three nonlinear constraints are transferred by the SecondOrder Cone Programming (SOCP) method (Alizadeh, Goldfarb 2003). Four positive variables, $v_{1}, v_{2}, v_{3}, v_{4}$, are introduced as auxiliary variables (Hu et al. 2014):

- the constraint $1 \leq q^{2} \cdot a^{5}$ equals to:

$$
\left\{v_{1}^{2} \leq a, v_{2}^{2} \leq v_{1} \cdot a, 1^{2} \leq a \cdot v_{2}\right\} \text {; }
$$

- the constraint $1 \leq q \cdot a^{3}$ equals to:

$$
\left\{v_{1}^{2} \leq a \cdot q, 1^{2} \leq a \cdot v_{1}\right\} ;
$$

- the constraint $1 \leq q^{2} \cdot a^{7}$ equals to:

$$
\left\{v_{1}^{2} \leq a, v_{2}^{2} \leq v_{1} \cdot q, v_{3}^{2} \leq v_{2} \cdot a, v_{4}^{2} \leq v_{3}, 1^{2} \leq v_{3} \cdot v_{4}\right\} .
$$

A hyperbolic inequality of the form $z^{2} \leq x \cdot y$ for non-negative values $(x, y, z \geq 0)$ can be rewrote as a SOCP constraint: $\|(2 \cdot z, x-y)\|_{2} \leq x+y$, because the constraint $z^{2} \leq x \cdot y$ is equivalent to the constraint $4 \cdot z^{2}+\left(x^{2}-2 \cdot x \cdot y+y^{2}\right) \leq\left(x^{2}+2 \cdot x \cdot y+y^{2}\right)$.

Therefore, for each $a_{i}$, five non-negative variables $\left(q_{i}\right.$, $\left.v_{i, 1}, v_{i, 2}, v_{i, 3}, v_{i, 4}\right)$ are denoted and the parts $a^{1-u_{i}}$, where $u_{i} \in\{3.5,4,4.5\}$ can be transformed to SOCP constraints as presented in Equations (32)-(34).

$$
\begin{aligned}
& \left.\begin{array}{l}
\left\|\left(2 v_{i, 1}, a_{i}-1\right)\right\|_{2} \leq a_{i}+1 \\
\left\|\left(2 v_{i, 2}, v_{i, 1}-q_{i}\right)\right\|_{2} \leq v_{i, 1}+q_{i} \\
\left\|\left(2, a_{i}-v_{i, 2}\right)\right\|_{2} \leq a_{i}+v_{i, 2}
\end{array}\right\}, \forall i \in V^{\text {Feeder }} ;
\end{aligned}
$$

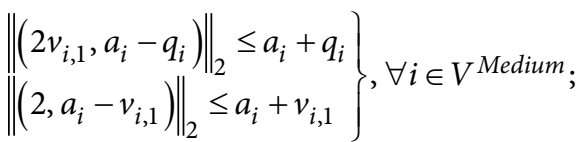

$$
\begin{aligned}
& \begin{array}{l}
\left\|\left(2 v_{i, 1}, a_{i}-1\right)\right\|_{2} \leq a_{i}+1 \\
\left\|\left(2 v_{i, 2}, v_{i, 2}-q_{i}\right)\right\|_{2} \leq v_{i, 1}+q_{i}
\end{array} \\
& \left.\left\|\left(2 v_{i, 3}, a_{i}-v_{i, 2}\right)\right\|_{2} \leq a_{i}+v_{i, 2}\right\}, \forall i \in V^{\text {Jumbo }} \text {. } \\
& \left\|\left(2 v_{i, 4}, v_{i, 1}-1\right)\right\|_{2} \leq v_{i, 1}+1 \\
& \left\|\left(2, v_{i, 3}-v_{i, 4}\right)\right\|_{2} \leq v_{i, 3}+v_{i, 4}
\end{aligned}
$$

\subsection{The multi-objective model}

A set of vessels considered in the berth plan is denoted by $V$, indexed by $i, j$. As mentioned above, $V$ is an union of $V^{\text {Feeder }}, V^{\text {Medium }}$ and $V^{\text {Jumbo }}$.

Five groups of parameters are known. $L$ denotes the length of quay side of the container terminal; $L_{i}$ denotes the length of vessel $i$; $T_{i}$ denotes the handling time of vessel $i ;\left[\underline{A}_{i}, \bar{A}_{i}\right]$ presents the range of EAT of vessel $i ; D_{i}$ is the requested departure time of vessel $i$; $M$ refers to a sufficiently large constant.

Five groups of decision variables are involved. The leftmost berthing position of vessel $i$ is denoted by $x_{i}$; the start time of berthing vessel $i$ is denoted by $t_{i}$; the arrival time of berthing vessel $i$ is $a_{i}$. If the vessel $i$ is scheduled at a left position of vessel $j$ along the quay side, $\sigma_{i j}^{X}=1$; otherwise, zero. If the vessel $i$ is scheduled to start earlier than vessel $j, \sigma_{i j}^{T}=1$; otherwise, zero.

The low-emission BAP is formulated as follows. The berthing position of each vessel is constrained as Equation (13). The vessel's arrival time and starting time of handling the vessel are constrained in Equations (14) and (15). For any two vessels scheduled, their schedules can not be overlapped in the tempo-space diagram, as constrained in Equations (16)-(18). 


$$
\begin{aligned}
& x_{i}+L_{i} \leq L, \forall i ; \\
& a_{i} \leq t_{i}, \forall i ; \\
& \underline{A}_{i} \leq a_{i} \leq \bar{A}_{i}, \forall i ; \\
& x_{i}+L_{i} \leq x_{j}+L \cdot\left(1-\sigma_{i j}^{X}\right), \forall i, j ; \\
& t_{i}+T_{i} \leq t_{j}+M \cdot\left(1-\sigma_{i j}^{T}\right), \forall i, j ; \\
& 1 \leq \sigma_{i j}^{X}+\sigma_{j i}^{X}+\sigma_{i j}^{T}+\sigma_{j i}^{T} \leq 2, \forall i, j .
\end{aligned}
$$

In this study, hard constraints on the berth and departure times are also formulated, as Equations (19) and (20). All vessels are scheduled in the $72 \mathrm{~h}$ horizon; they should be berthed within the $72 \mathrm{~h}$ (Equation (20)). A schedule of $72 \mathrm{~h}$ is used here for the following reasons. First, the mathematical programs are developed to solve the problem while the computing time will be unacceptable for the schedule for many days. Second, in terminal operations, the minor adjustments about berthing or departure times will be made according to the timely (usually one day or two days) reports from the vessels. A new control parameter $\bar{D}$ is used to represent the maximal delay $D_{i}$ compared to the EDT. This parameter is usually related to a service quality degree promised by the terminal operators.

$$
\begin{aligned}
& t_{i} \leq 72, \forall i ; \\
& t_{i}+T_{i} \leq D_{i}+\bar{D}, \forall i .
\end{aligned}
$$

The types of variables are set in Equation (21), $a_{i}, t_{i}, x_{i}$ are positive and continuous, and $\sigma_{i j}^{X}$ and $\sigma_{i j}^{T}$ are integers:

$$
a_{i}, t_{i}, x_{i} \geq 0, \sigma_{i j}^{X} \in\{0,1\}, \sigma_{i j}^{T} \in\{0,1\}, \forall i, j .
$$

Three objectives are considered for evaluating the service quality degree $z^{T}$, emissions in the sailing $z^{\text {ESail }}$ and mooring $z^{\text {EMoor }}$ periods in Equations (22)-(29):

- in Equation (22), the delayed time compared to the EDT of each vessel is formulated;

- the emission of vessel $i$ when sailing is denoted by $z_{i}^{\text {ESail }}$, which is computed by Equation (5), namely $e_{i}^{\text {Sail }}=f_{i} \cdot E^{n S a i l}$. The fuel consumption of the vessel is computed by Equation (1), namely $f_{i}=\lambda_{i}^{0} \cdot a_{i}+\lambda_{i}^{1} \cdot M_{i}^{u_{i}} \cdot a_{i}^{1-u_{i}}$. Then, $z_{i}^{\text {ESail }}$ is derived by Equation (23). Notably $a_{i}^{1-u_{i}}$ is nonlinear when the arrival times are decision variables here. We introduce Equation (24) to transfer Equation (23) to a linear Equation (25). Because the sailing emission should be minimized in the model, the inequality relation is used in Equation (24). The inequality $a_{i}^{1-u_{i}} \leq q_{i}$ can be further transferred to $1 \leq a_{i}^{u_{i}-1} \cdot q_{i}$. Considering the candidate values of $u_{i}\left(u_{i} \in\{3.5,4,4.5\}\right)$, it can be transferred to SOCP constraints, as presented in Equations (10)-(12).

$$
\begin{aligned}
& z_{i}^{T}=\left(t_{i}+T_{i}-D_{i}\right)^{+}=\max \left(t_{i}+T_{i}-D_{i}, 0\right), \forall i ; \\
& z_{i}^{\text {ESail }}=f_{i} \cdot E^{n S a i l}=\left(\lambda_{i}^{0} \cdot a_{i}+\lambda_{i}^{1} \cdot M_{i}^{u_{i}} \cdot a_{i}^{1-u_{i}}\right) \cdot E^{n S a i l} ;
\end{aligned}
$$

$$
\begin{aligned}
& a_{i}^{1-u_{i}} \leq q_{i}, \forall i ; \\
& z_{i}^{\text {ESail }}=\left(\lambda_{i}^{0} \cdot a_{i}+\lambda_{i}^{1} \cdot M_{i}^{u_{i}} \cdot q_{i}\right) \cdot E^{n S a i l} ;
\end{aligned}
$$

- the emission of vessel $i$ when mooring is denoted by $z_{i}^{E M o o r}$, which is computed by Equation (9). Here, $t_{i}^{a c t i v e}$ is represented by the waiting and handling time at the terminal, namely $z_{i}^{T}$. Therefore, based on Equation (26), $z_{i}^{\text {EMoor }}$ is computed by Equation (27):

$$
\begin{aligned}
& z_{i}^{\text {EMoor }}=e_{i}^{\text {Moor }}=t_{i}^{\text {active }} \cdot E_{i}^{\text {Moor }} ; \\
& z_{i}^{\text {EMoor }}=z_{i}^{T} \cdot E_{i}^{\text {Moor }} .
\end{aligned}
$$

Based on the above derivations of the three objectives related to each vessel, the objectives are finally formulated by Equations (28)-(30):

$$
\begin{aligned}
& z^{T}=\sum_{i} T_{i} \cdot z_{i}^{T} ; \\
& z^{\text {ESail }}=\sum_{i} z_{i}^{\text {ESail }} ; \\
& z^{\text {EMoor }}=\sum_{i} z_{i}^{\text {EMoor }} .
\end{aligned}
$$

To minimize these three objectives, the model $[P 1]$ is formulated as follows. The complete list of objectives and constraints are listed in the Appendix.

$[P 1]$ :

$\min z=\left(z^{T}, z^{E S a i l}, z^{E M o o r}\right)$

subject to:

Equations (10)-(22), (25), (27)-(30);

$q_{i}, v_{i, 1}, v_{i, 2}, v_{i, 3}, v_{i, 4} \geq 0, \forall i$.

Notably, five sets of new variables are introduced for transferring the nonlinear constraints to the SOCP constraints. So the complete vector of variables is $\mathbf{x}$ :

$$
\mathbf{x}=\left(x_{i}, a_{i}, t_{i}, \sigma_{i j}^{X}, \sigma_{i j}^{Y}, q_{i}, v_{i, 1}, v_{i, 2}, v_{i, 3}, v_{i, 4}\right) .
$$

Notably, $z_{i}^{T}, z_{i}^{E M o o r}, z_{i}^{E S a i l}$ are three sets of auxiliary variables used for analysis.

The complete known parameters used in $[P 1]$ is $\mathbf{P}$ :

$$
\mathbf{P}=\left(L, L_{i}, \underline{A}_{i}, \bar{A}_{i}, D_{i}, \bar{D}, T_{i}, M_{i}, \lambda_{i}^{0}, \lambda_{i}^{1}, E_{i}^{\text {Moor }}, E^{n S a i l}\right) .
$$

The effects of sailing and mooring emissions on environment can be considered together by Equation (31). Here, $\alpha$ is a parameter to control the preference of the emission in the scenario of mooring periods comparing to the sailing emission. Although the two types of emissions are formulated in $[P 1]$ (see the complete constraints and objectives in the Appendix) by using the same measures, generally the mooring emission imposes greater effects on the environment and human because the terminals are usually near the port cities.

$$
z^{E}=z^{\text {ESail }}+\alpha \cdot z^{\text {EMoor }} \text {. }
$$

Four objectives $\left(z^{T}, z^{\text {ESail }}, z^{\text {EMoor }}, z^{E}\right)$ can be solely minimized by $[P 2]$. The minimums are denoted by $\underline{z}^{T}$, $\underline{z}^{\text {ESail }}, \underline{z}^{\text {EMoor }}, \underline{z}^{E}$, individually: 


$$
\begin{aligned}
& \underline{z}^{T}=\min \left(z^{T} \mid[P 1]\right) ; \\
& \underline{z}^{\text {ESail }}=\min \left(z^{\text {ESail }} \mid[P 1]\right) ; \\
& \underline{z}^{\text {EMoor }}=\min \left(z^{\text {EMoor }} \mid[P 1]\right) ; \\
& \underline{z}^{E}=\min \left(z^{E} \mid[P 1], \text { Equation }(31)\right) .
\end{aligned}
$$

In $[P 2],[P 3]$ and $[P 4]$, the bi-objective models are solved by the method based on $\varepsilon$-constraint. In the $\varepsilon$-constraint method we optimize one of the objective functions using the other objective functions as constraints, incorporating them in the constraint part of the model (Mavrotas 2009). Here, $\varepsilon^{E S a i l}, \varepsilon^{E}$ and $\varepsilon^{T}$ are relaxation degrees comparing the minimums $\left(\underline{z}^{\text {ESail }}, \underline{z}^{E}\right.$ and $\left.\underline{z}^{T}\right)$. Different from $[P 2]-[P 4],[P 5]$ is used to investigate the effect of minimizing the mooring emission on the solutions of minimizing the sailing emission while the optimal service quality degree is met, while $[P 6]$ is used to examine the effect of minimizing the service quality degree on the solutions of minimizing the sailing emission while the optimal mooring emission is met.

$[P 2]:$

$\min \left(z^{T} \mid z^{E S a i l} \leq\left(1+\varepsilon^{E S a i l}\right) \underline{z}^{\text {ESail }},[P 1]\right.$, Equation (31));

$[P 3]:$

$\min \left(z^{T} \mid z^{E} \leq\left(1+\varepsilon^{E}\right) \underline{z}^{E},[P 1]\right.$, Equation (31));

$[P 4]$ :

$\min \left(z^{\text {EMoor }} \mid z^{T} \leq\left(1+\varepsilon^{T}\right) \underline{z}^{T}, z^{\text {ESail }} \leq\right.$

$\left(1+\varepsilon^{E S a i l}\right) \underline{z}^{E S a i l},[P 1]$, Equation (31));

$[P 5]$ :

$\min z^{\text {ESail }}=\left(z^{\text {ESail }} \mid z^{T} \leq \underline{z}^{T}, z^{\text {EMoor }} \leq z^{\text {EMoor }}=\right.$

$\left.\min \left(z^{\text {EMoor }} \mid z^{T} \leq \underline{z}^{T},[\mathrm{P} 1]\right),[\mathrm{P} 1]\right)$;

$[P 6]$ :

$\min z^{\text {ESail }}=\left(z^{\text {ESail }} \mid z^{\text {EMoor }} \leq \underline{z}^{\text {EMoor }}, z^{T} \leq z^{T}=\right.$

$\left.\min \left(z^{T} \mid z^{\text {EMoor }} \leq \underline{z}^{\text {EMoor }},[\mathrm{P} 1]\right),[\mathrm{P} 1]\right)$.

\section{Data estimation and test data generation}

Table 1 presents their reference values of the emission factors while sailing (COSCO 2009), where $E^{n \text { Sail }}$ is computed by the following formula:

$$
E^{n \text { Sail }}=\sum_{n} E_{n}^{\text {Sail }}=3110+87+60=3257 .
$$

As emissions from auxiliary engines dominate while berthed, this work evaluates emissions while berthed from auxiliary engines. Table 2 presents reference values of emission factors while mooring. Moreover, this work sets $E^{L F}=0.5$ according to average power data for auxiliary engines (Starcrest Consulting Group 2011), and sets $E_{i}^{\text {Engine }}=4$ because most sea-going vessels have 4 -stroke auxiliary engines. The notation $E^{n M o o r}$ is introduced and computed by the following formula:

$$
E^{n M o o r}=\sum_{n} E\left(\begin{array}{l}
\text { Moor } \\
n
\end{array} E_{n}^{\text {Fuel }}\right)=680 \cdot 1+13 \cdot 0.948+
$$$$
12.3 \cdot 0.04=692.816 \text {. }
$$

The data related to BAP from Ningbo Beilun Port is used to generate the parameters of the models. A quay (1200 m long) and a planning horizon of $72 \mathrm{~h}$ are considered. The wharf is quantified by a unit of $50 \mathrm{~m}$ (WU), and the unit of time of the planning horizon is one hour (TU). Therefore, $L=1200 / 50=24$. Some related parameters are generated randomly from uniform distributions whose lower and upper bounds are defined by intervals (Table 3). Other parameters are generated by: $A_{i} \sim U[0,62]$, $T_{i} \sim U[9,35], D_{i} \sim T_{i} \cdot U[1,2]+A_{i}, M_{i}=A_{i} \cdot s_{i}^{0}$, where: $s_{i}^{0}$ is the initial sailing speed of vessel $i$ at the beginning of a berthing plan. For a feeder vessel $i$, set $\underline{A}_{i}=M_{i} / 24$ and $\bar{A}_{i}=M_{i} / 10$; for medium-sized vessel $i$, set $\underline{A}_{i}=M_{i} / 28$ and $\bar{A}_{i}=M_{i} / 12$; for jumbo vessel $i$, set $\underline{A}_{i}=\bar{M}_{i} / 30$ and $\bar{A}_{i}=M_{i} / 14$. In the experiments, $30 \%$ of vessels are in the feeder class, $50 \%$ of vessels are in the medium-size class, and $20 \%$ of vessels are in the jumbo class.

By the above settings, a list of 30 vessels is generated as the data set used in the experiments in Section 5.

Table 1. Reference values of emission factors while sailing

\begin{tabular}{|l|c|c|c|}
\hline Emission factor $n$ & $1\left(\mathrm{CO}_{2}\right)$ & $2\left(\mathrm{NO}_{\mathrm{x}}\right)$ & $3\left(\mathrm{SO}_{\mathrm{x}}\right)$ \\
\hline Emissions $E_{\text {in }}^{\text {Sail }}[\mathrm{g} / \mathrm{kg}$ fuel $]$ & 3110 & 87 & 60 \\
\hline
\end{tabular}

Table 2. Reference values of emission factors while mooring

\begin{tabular}{|l|c|c|c|}
\hline Emission factor $n$ & $1\left(\mathrm{CO}_{2}\right)$ & $2\left(\mathrm{NO}_{\mathrm{x}}\right)$ & $3\left(\mathrm{SO}_{\mathrm{x}}\right)$ \\
\hline Emissions $E_{\text {in }}^{\text {Moor }}[\mathrm{g} / \mathrm{kW} \cdot \mathrm{h}]$ & 683 & 13 & 12.3 \\
\hline Fuel correction factors $E_{n}^{\text {Fuel }}$ & 1 & 0.948 & 0.04 \\
\hline Load ratio $E^{L F}$ & 0.5 & - & - \\
\hline $\begin{array}{l}\text { Number of engines } \\
\text { of } i \text { vessel } E_{i}^{\text {Engine }}\end{array}$ & 4 & - & - \\
\hline
\end{tabular}

Table 3. Parameters of uniform distribution for three vessel classes

\begin{tabular}{|l|c|c|c|c|c|c|}
\hline \multicolumn{1}{|c|}{ Class } & $L_{i}[\mathrm{WU}]$ & $\lambda_{i}^{0}$ & $\lambda_{i}^{1}\left[\cdot 10^{-4}\right]$ & $s_{i}^{0}[\mathrm{knots}]$ & $E_{i}^{P O}[$ horsepower $]$ & $u_{i}$ \\
\hline Feeder & {$[1,4)$} & {$[477.4,719.9]$} & {$[151,245]$} & {$[10,24]$} & {$[50,100)$} & 3.5 \\
\hline Medium & {$[4,6]$} & {$[580.7,718.6]$} & {$[37.09,42.99]$} & {$[12,28]$} & {$[100,250)$} & 4.0 \\
\hline Jumbo & $(6,8]$ & {$[491.7,709.2]$} & {$[8.64,9.72]$} & {$[14,30]$} & {$[250,425]$} & 4.5 \\
\hline
\end{tabular}




\section{Numerical experiments}

The models are formulated in a MATLAB environment, (https://www.mathworks.com) and solved by a Gurobi 6.0 (https://www.gurobi.com). All numerical experiments are performed on a personal computer with an Intel Core i5 $2.67 \mathrm{GHz}$ CPU and $8 \mathrm{~GB}$ RAM. Due to the complexity of port operational cost and fuel consumption, and the effects of various parameters, four groups of experiments are used to assess the applicability of the proposed methods under various operational conditions.

\subsection{Demonstrating the minimization of delay time or emissions}

The values of four objectives $\left(z^{T}, z^{E S a i l}, z^{E M o o r}\right.$ and $\left.z^{E}\right)$, when one of them is minimized solely are listed in Table 4 . In the instance, the weighted delay time can be almost zero, when $z^{T}, z^{E M o o r}$ or $z^{E}$ is solely minimized. The schedules corresponding to these four executions are presented in Figure 3, where the dark grey boxes indicate the delayed vessels. In other words, for this instance, minimizing $z^{T}$ is not conflict with minimizing $z^{E M o o r}$ and $z^{E}$. With the present parameters, the weighted delay time can reach zero (Table 4). When the sailing emission is minimized, the weighted delay time is 817 (Table 4) and 8 vessels are delayed comparing to their EDTs (Figure $3 \mathrm{~b}$ ). The mooring emission is far less than the sailing emission. However, the mooring emission directly affects the port city. When the total emission $z^{E}$ is minimized, the mooring emission can be prominently minimized comparing to the sailing emission. Therefore, just considering the weighted delay time and the total emission is promising in reducing emission. In Table 4, the sailing emission can only be reduced by $0.02 \%$ from 1266711157 to 1266440827 , while the mooring emission can be reduced by 29029074 to zero.

Based on the above results, the experiments are conducted in the following. First, the trade-offs between minimizing $z^{T}$ and $z^{E S a i l}$ are examined. Whether do the tradeoffs exist and how about their degrees are investigated in the Section 5.2. Second, the effects of the key parameters, vessels' handling times $T_{i}$ and EDTs $\bar{D}_{i}$ on the solutions are studied in the Section 5.3. Third, the reduced emissions may be increased when more vessels are scheduled together, which is checked in the Section 5.4.

\subsection{Minimizing emissions and weighted delay times}

As presented in Table 4, minimizing $z^{T}$ and $z^{E S a i l}$ is possibly conflict because $z^{T}$ is bigger when $z^{E S a i l}$ is minimized. To test the possibility of conflict between these two objectives, $[P 2]$ is solved for the case when $\varepsilon^{E S a i l}$ is set to zero. The results are presented in Figure 4a. By the same process, $[P 3]$ is solved resulting in Figure $4 \mathrm{~b}$ when $\varepsilon^{E}$ is set to zero. Comparing to Figure $3 \mathrm{~b}$ and $3 \mathrm{~d}$, the weighted delay times in Figure 4 also reaches zero while the minimum of $z^{E S a i l}$ or $z^{E}$ is also guaranteed.

The values of sailing and mooring emissions in Table 4 show that they may be conflict with each other. $[P 4]$ is used here to test whether the conflict relation exits, when $\varepsilon^{E S a i l}$ and $\varepsilon^{T}$ are set to zeroes. The results are presented in Figure 5.

We can conclude the above experiments that the emissions and delays can be simultaneously minimized. Because the vessels' arrival and departure times are flexible within limited ranges, the four objectives may be not strictly conflict. Therefore, in the following experiments, three strategy is used: (1) we minimized the weighted delays; (2) the minimal mooring emission is computed with the minimal delay; (3) the sailing emission is minimized when the above minimal delay and mooring emission are pursued. Why is this tri-stage optimization strategy used? The above experiments show that the delay can be not conflict with the emission minimization purpose. Although the mooring emission is minor comparing to the sailing emission, it affects the port cities' environments directly. This strategy is entitled as Time Mooring Sailing (TMS) strategy and presented in [P5]. Similarly, the Mooring Time Sailing (MTS) strategy can be developed as presented by $[P 6]$.

\subsection{Effects of handling times and EDTs on emissions}

The handling times $T_{i}$ of vessels reflect the efficiency of the terminal and the costs paid by the terminal operators. The EDTs $\bar{D}_{i}$ of vessels affect the vessels' chartering costs. Here, $\bar{D}_{i}$ is transferred to $\tilde{D}_{i}$ as relative values, $\tilde{D}_{i}=\left(\bar{D}_{i}-T_{i}-\underline{A}_{i}\right)$. In the proposed models, these two parameters affect the interests of terminal operators and shipping companies. To study the effects of $T_{i}$ and $\bar{D}_{i}$ (represented by $\tilde{D}_{i}$ ) on the solutions, the values of them

Table 4 . The four objectives of solely minimizing one of them

\begin{tabular}{|l|c|c|c|c|}
\hline \multicolumn{1}{|c|}{ Model } & $z^{T}[\mathrm{~h}]$ & $z^{\text {ESail }[\mathrm{g}]}$ & $z^{\text {EMoor }}[\mathrm{g}]$ & $z^{E}[\mathrm{~g}]$ \\
\hline$\underline{z}^{T}=\min \left(z^{T}[[M 1])\right.$ & $\underline{0}$ & 1266711157 & 0 & 1266711157 \\
\hline$\underline{z}^{\text {ESail }}=\min \left(z^{\text {ESail }}[[M 1])\right.$ & 817 & $\underline{1266440827}$ & 29029074 & 1295469901 \\
\hline$\underline{z}^{\text {EMoor }}=\min \left(z^{\text {EMoor }}[[M 1])\right.$ & 0 & 1266711157 & $\underline{0}$ & 1266711157 \\
\hline$\underline{z}^{E}=\min \left(z^{E}[\right.$ M1],Equation (31) $)$ & 9 & 1266440827 & 103923 & $\underline{1266544750}$ \\
\hline
\end{tabular}


a)

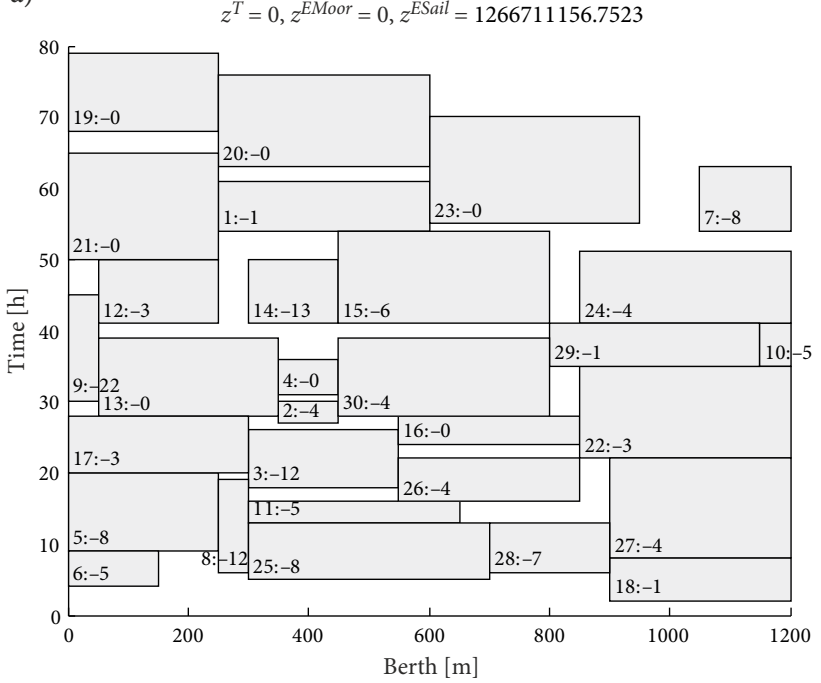

c)

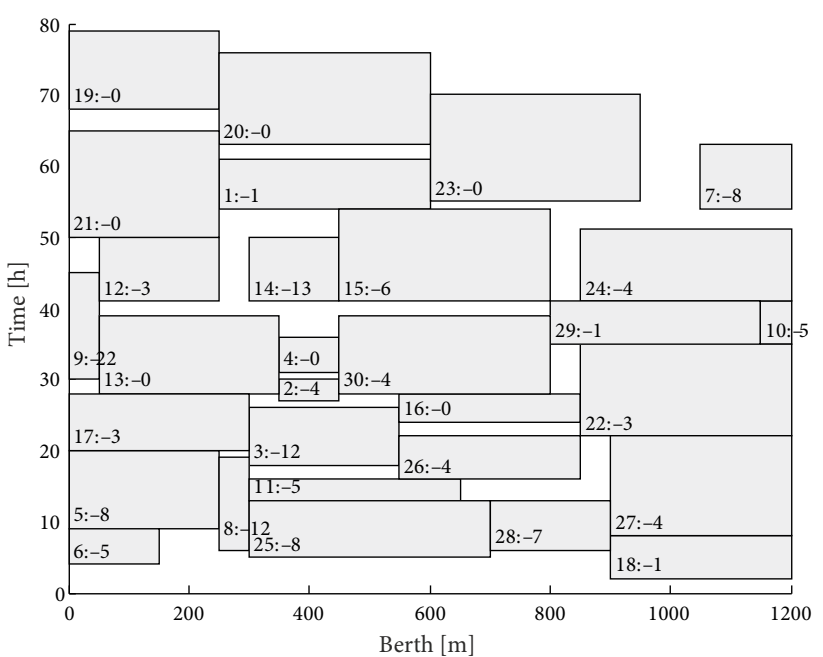

b)

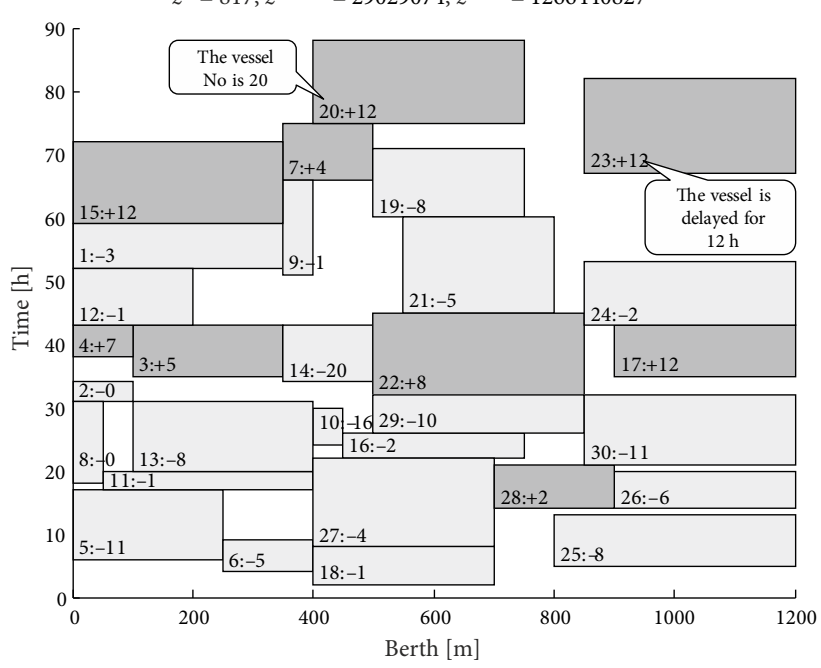

d)

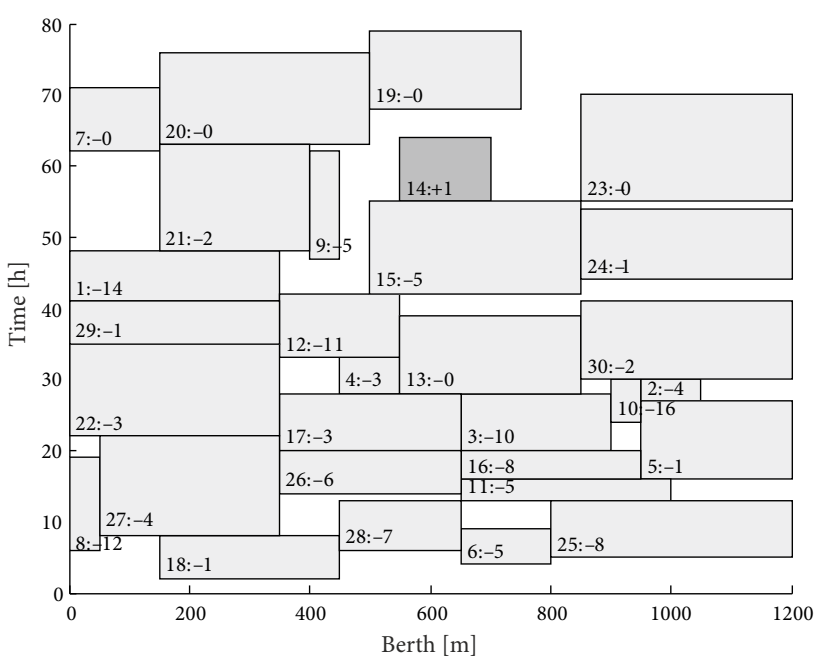

Figure 3. The schedules of solely minimizing one of four objectives: a - weighted delay time; $\mathrm{b}$ - sailing emission; $\mathrm{c}$ - mooring emission; $\mathrm{d}$ - emission

a)

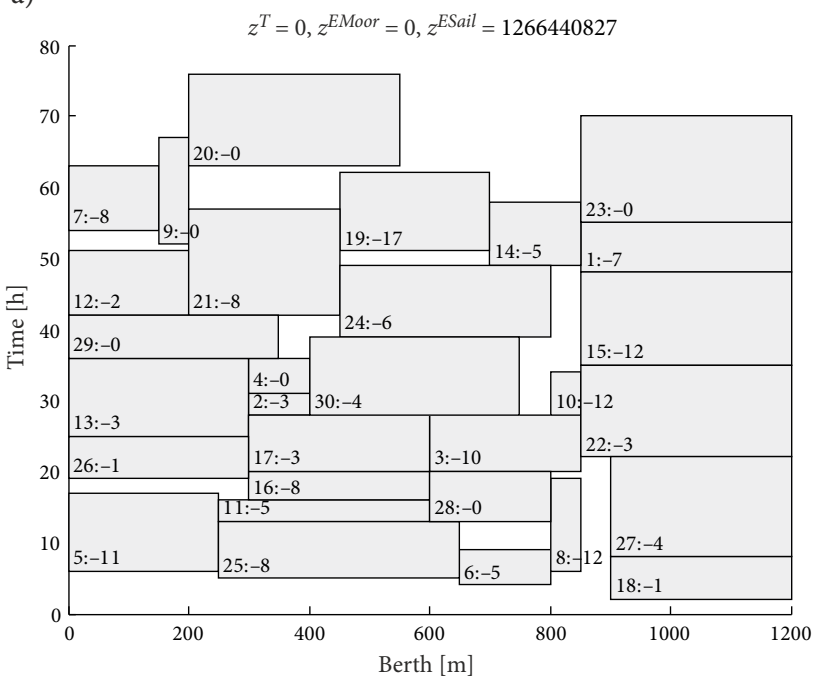

b)

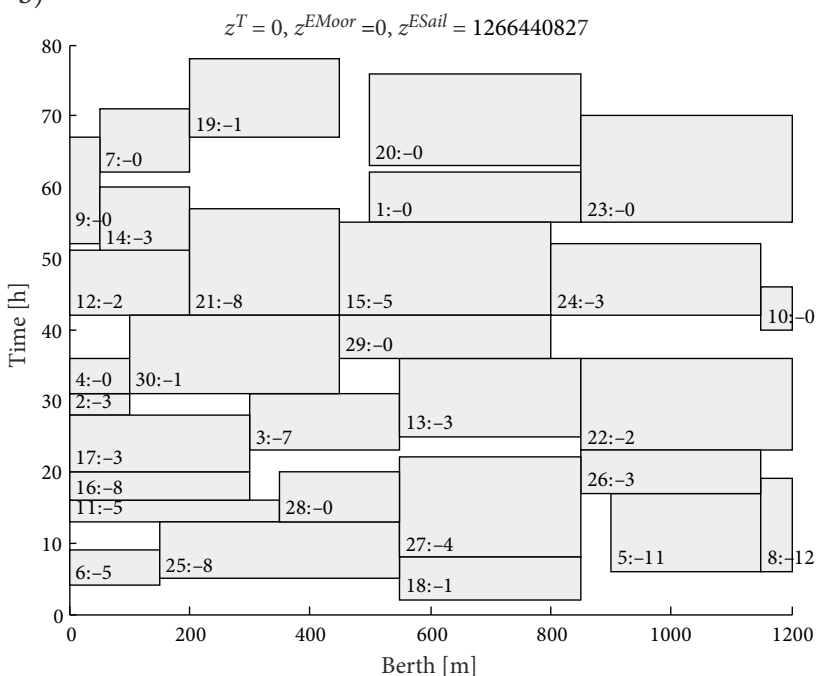

Figure 4. Minimizing emission and then weighted delay by two-stage method: a - minimizing sailing emission and then weighted delay; $\mathrm{b}$ - minimizing total emission and then weighted delay 


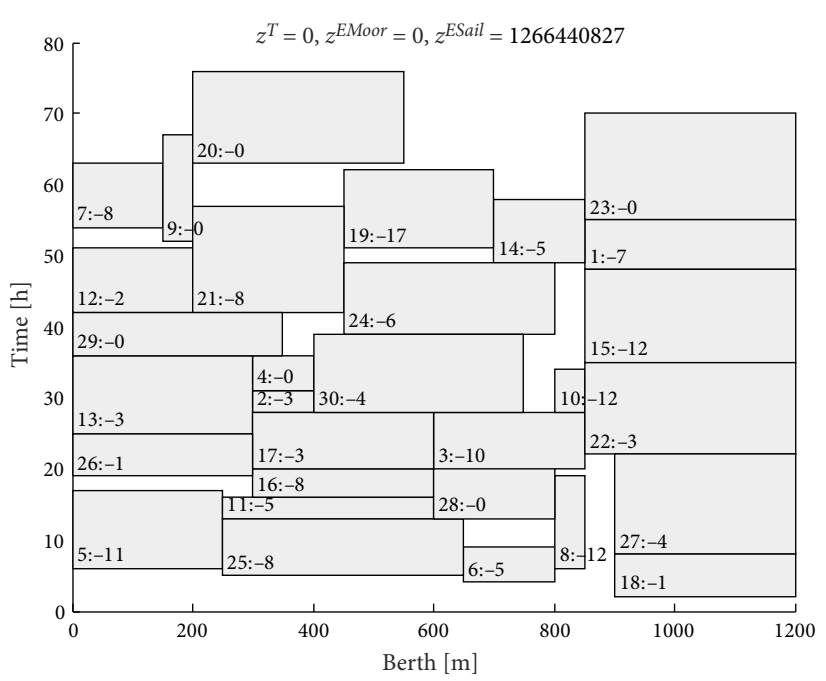

Figure 5. Minimizing mooring emission for zero delay and minimal sailing emission

are varied $\left(T_{i} \leftarrow \tau \cdot T_{i}\right.$, and $\left.\tilde{D}_{i} \leftarrow \xi \cdot \tilde{D}_{i}\right)$, and the TMS and MTS strategies are used to solve the models, $[P 5]$ and $[P 6]$. Here, $\tau$ and $\xi$ are values in $0.1,0.2, \ldots, 2$. The results are presented in the Tables 5 and 6 .

In Table 5, under the TMS strategy, with the decrease of the vessels' handling times from the reference values $(\tau=1)$, the solution objectives are not affected. However, when they are increased to 1.1 or 1.2 times, the vessels will be seriously delayed while the sailing emissions are not changed. Under the MTS strategy, the results are similar. When the handling times increase more, feasible results can not be found within $10 \mathrm{~min}$.
The EDT values determine the ranges of berth times. Therefore, when they are relaxed, the delay times and mooring emissions should be reduced. Inversely, when they are decreased, delay times and mooring emissions may rise. As presented in Table 6, we also can see that the mooring emissions increase when we minimize the delay times first, and the weighted delay times increase when we minimize the mooring emission first. When at least 0.9 times of EDTs are set, the delay times and mooring emission are zeroes.

\subsection{Effects of number of vessels on reducing emissions}

The dataset as the same as the one used above is used to demonstrate the effects of the number of vessels on emission reduction. The time-emission strategy is used here, as presented in $[P 6]$ : the minimal weighted delay time $z^{T}$ is computed first by Equation (32), resulting with a value of emission $\bar{z}^{E}$; and then the weighted delay time is constrained by this minimum and the minimal emission $z^{E}$ is optimized, which is denoted by $z^{E}$. In the experiment, the number of vessels is increased from 10 to 30 according to their EATs $A_{i}$. For each time, $[P 6]$ is applied to a dataset and the reduced emission can be computed by $\left(\bar{z}^{E}-\breve{z}^{E}\right)$ . Two experiments are conducted here. First, the dataset used above is used by adding a new vessel into the vessel set from 10 to 30 vessels. So, 21 groups of results were obtained. Second, forty datasets are generated according to the data generation rules set in the Section 4. The steps used in the first experiment are repeated for each of these datasets. And then average emission is computed for each

Table 5. The objectives affected by $T_{i}$ under the TMS and MTS strategies

\begin{tabular}{|c|c|c|c|c|c|c|}
\hline \multirow{2}{*}{$\tau$} & \multicolumn{3}{|c|}{ TMS } & \multicolumn{2}{c|}{ MTS } \\
\cline { 2 - 7 } & $\underline{z}^{T}$ & $z^{\text {EMoor }}$ & $z^{\text {ESail }}$ & $\underline{z}^{T}$ & $\underline{z}^{\text {EMoor }}$ & $z^{\text {ESail }}$ \\
\hline $0.1 \ldots 1.0$ & 0 & 0 & 1266440827 & 0 & 0 & 1266440827 \\
\hline 1.1 & 228.47 & 8280907 & 1266440827 & 219.45 & 7033835 & 1266440827 \\
\hline 1.2 & 851.76 & 26867482 & 1266440827 & 859.68 & 0 & 1266440827 \\
\hline $1.3 \ldots 2.0$ & - & - & - & - & - & - \\
\hline
\end{tabular}

Table 6. The objectives affected by $\bar{D}_{i}$ under the TMS and MTS strategies

\begin{tabular}{|c|c|c|c|c|c|c|}
\hline \multirow{2}{*}{$\xi$} & \multicolumn{3}{|c|}{ TMS } & \multicolumn{2}{c|}{ MTS } \\
\cline { 2 - 7 } & $\underline{z}^{T}$ & $z^{\text {EMoor }}$ & $z^{\text {ESail }}$ & $\underline{z}^{\text {EMoor }}$ & $\underline{z}^{\text {ESail }}$ \\
\hline 0.1 & 0.0 & 41792514 & 1266440827 & 1317.5 & 0 & 1266440827 \\
\hline 0.2 & 986.4 & 36137387 & 1266440827 & 1160.0 & 35125873 & 1266440827 \\
\hline 0.3 & 704.8 & 26619800 & 1266440827 & 782.5 & 24311821 & 1266440827 \\
\hline 0.4 & 527.2 & 22229066 & 1266440827 & 535.4 & 21408076 & 1266440827 \\
\hline 0.5 & 296.0 & 13059619 & 1266440827 & 411.0 & 12721871 & 1266440827 \\
\hline 0.6 & 92.2 & 7787274 & 1266440827 & 279.4 & 6595627 & 1266440827 \\
\hline 0.7 & 56.2 & 5634342 & 1266440827 & 73.2 & 140296 & 1266440827 \\
\hline 0.8 & 0 & 210127 & 1266440827 & 64.6 & 0 & 1266440827 \\
\hline $0.9 \ldots 2$ & 0 & 0 & 1266440827 & 0.0 & 0 & 1266440827 \\
\hline
\end{tabular}


a)

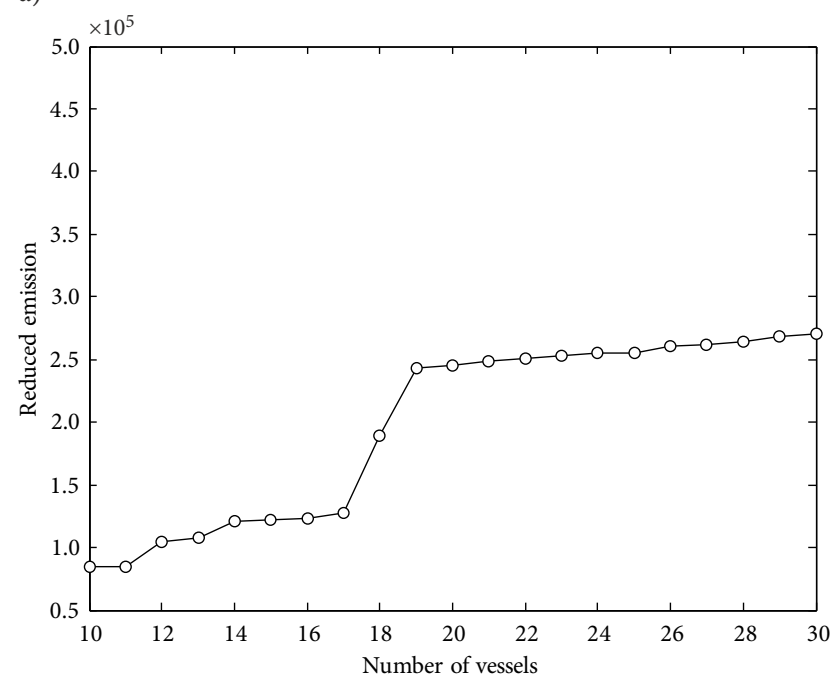

b)

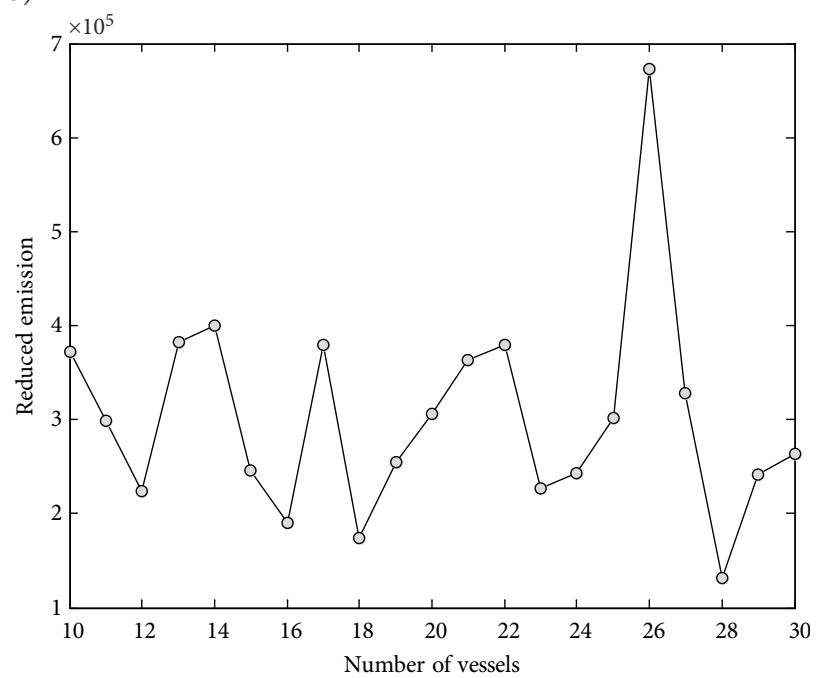

Figure 6. Reduced emissions varying with number of vessels: $a$ - a sample; b - average reduced emissions

setting of number of vessels. The results for the two experiments are presented by Figure 6.

In Figure $6 a$, the reduced emission rises generally. In Figure $6 \mathrm{~b}$, the reduced emissions fluctuate with the number of vessels irregularly. For a given dataset, when new vessels are added into the vessel set for optimization, the reduced emission will increase. The purpose of the second experiment is to verify whether a general trend does also exist. Obviously, from Figure 6b, the resulting curve is irregular, possibly because the results are so sensitive to the time and space occupied by the handled vessels. In this study, the time demanded for handling a vessel is given by known data. When it can be variables, the results should be different. However, new formulations and algorithms are to be developed for such experiments.

\section{Conclusions}

This work developed a multi-objective model for the BAP by considering the weighted delay time comparing to the EDTs, the emission during the sailing periods, and the emission during the mooring periods when the vessels call for service at container terminals. The sailing speeds and mooring times determine the sailing and mooring emissions, and their varying ranges are affected by the arrival times and berth times at the terminals. These values that are generally known parameters in general BAPs are decision variables in the developed models. To transform the nonlinear emission functions of the sailing speeds to linear ones, the SOCP technique is used. So the proposed model can be solved by SOCP-compatible solvers. To reveal the trade-offs or other special relations among the delay times and emissions, a series of models are derived and numerically analysed. By merging the sailing and mooring emissions into a single objective, the tri-objective optimization model (weighted delay time, sailing and mooring emissions) can be transferred into a bi-objective model (weighted delay time, emissions), which can help simplify the computation complexity and is promising in reducing the emissions, especially the mooring emission. To investigate the effects of some key parameters (e.g., vessels' handling times, EDTs) on the solutions, their sensitivities are examined. To handle the computation complexity of the models and obtain sophisticated results, new algorithms should be developed for solving large-scale instances.

By four groups of experiments, the managerial implications are summarized here. First, the efficiency purpose is not conflict with the environment purposes for some instances. They can be pursued at the same time. Second, improving the vessel handling efficiency helps expand the ranges of berth times and sailing speeds. So the weight delay times and emission can be reduced. Apparently, the efficiency improvement indicates additional operational costs paid by the terminal operators. Advancing the EDTs involves the terminal operators' service quality to shipping companies, while the weighted delay times and emission may be increased. Therefore, the vessel handling efficiency and EDTs should be optimized by considering the interests of terminal operators and shipping companies. Third, the scheduling optimality should be increased by considering more vessels in the schedule. However, the delay times and emissions are directly affected by the berth times, which are further affected by the vessels' handling times and EDTs. In this study, the handling times are fixed for vessels, which decrease the possibility of decreasing the handling times. So the possible ranges of berth times are limited.

In terms of contributions, this work focused on analysing the relations among vessels' weighted delay times, sailing and mooring emissions, and the effects of key parameters on the solutions by extending the research conducted by Du et al. (2011) and Hu et al. (2014). This work directly investigated them by $\varepsilon$-constraint and stage-based formulations. The above managerial implications are derived from the experiments, which are also new in the research on BAPs. 
However, as mentioned above, the vessels' handling times and EDTs are fixed in this work. These two groups of parameters affect the adjustable ranges of berth times. The vessel's handling time may be reduced by allocating more quay cranes and related operational resources to the vessel. The EDT can be adjusted by coordinating the outbound container logistics and the terminals called by the vessel. In this study, the decision-making models are developed for terminal operators. However, the berth times at the terminal are dependent on the shipping companies' decisions and the berth allocation schedules of the other terminals called by the vessels. Therefore, these stakeholders should be considered in fine ways. So, as a practical direction, the coordination strategy should be researched and developed for the stakeholders for cooperatively reducing the emissions. We also try to persuade the terminal operators and shipping companies to accept the proposed methods as a way to green port and shipping. As another important issue, the distances from the sailing vessels to the terminals are important factors affecting the emissions and the impacts of emissions to the environments (Svindland 2018). As an interesting strategy, the sea area can be divided into regions (e.g., ECA) with different emission impacts (Fagerholt, Psaraftis 2015). On similar way, the aircraft emissions have been divided on the emissions below $3000 \mathrm{ft}$ (with airport emissions) and over $3000 \mathrm{ft}$ (with airplane emissions) (Masiol, Harrison 2014). New formulations and solution methods will be developed based on Corbett and Fischbeck (1997); Eyring et al. (2010) and Browning et al. (2010).

\section{Acknowledgements}

This study is partially supported by the National Natural Science Foundation of China (Grant No 71871136).

\section{Disclosure statement}

This study has not got any competing financial, professional, or personal interest from other parties.

\section{Appendix}

$[P 1]$ :

$\min z=\left(z^{T}, z^{E S a i l}, z^{E M o o r}\right)$,

where:

$$
\begin{aligned}
& z^{T}=\sum_{i} T_{i} \cdot z_{i}^{T} ; \\
& z^{\text {ESail }}=\sum_{i} z_{i}^{\text {ESail }} ; \\
& z^{\text {EMoor }}=\sum_{i} z_{i}^{\text {EMoor }},
\end{aligned}
$$

subject to:

$z_{i}^{T}=\left(t_{i}+T_{i}-D_{i}\right)^{+}=\max \left(t_{i}+T_{i}-D_{i}, 0\right)$,

$\forall i$;

(Equation 22)

$$
\begin{aligned}
& z_{i}^{\text {ESail }}=\left(\lambda_{i}^{0} \cdot a_{i}+\lambda_{i}^{1} \cdot M_{i}^{u_{i}} \cdot q_{i}\right) \cdot E^{n S a i l} ; \\
& z_{i}^{\text {EMoor }}=z_{i}^{T} \cdot E_{i}^{\text {Moor }} \text {; } \\
& x_{i}+L_{i} \leq L, \forall i \text {; } \\
& a_{i} \leq t_{i}, \forall i ; \\
& \underline{A}_{i} \leq a_{i} \leq \bar{A}_{i}, \forall i \text {; } \\
& x_{i}+L_{i} \leq x_{j}+L \cdot\left(1-\sigma_{i j}^{X}\right), \forall i, j ; \\
& t_{i}+T_{i} \leq t_{j}+M \cdot\left(1-\sigma_{i j}^{T}\right), \forall i, j ; \\
& 1 \leq \sigma_{i j}^{X}+\sigma_{j i}^{X}+\sigma_{i j}^{T}+\sigma_{j i}^{T} \leq 2, \forall i, j ; \\
& t_{i} \leq 72, \forall i \text {; } \\
& t_{i}+T_{i} \leq D_{i}+\bar{D}, \forall i ; \\
& \left.\begin{array}{l}
\left\|\left(2 v_{i, 1}, a_{i}-1\right)\right\|_{2} \leq a_{i}+1 \\
\left\|\left(2 v_{i, 2}, v_{i, 1}-q_{i}\right)\right\|_{2} \leq v_{i, 1}+q_{i} \\
\left\|\left(2, a_{i}-v_{i, 2}\right)\right\|_{2} \leq a_{i}+v_{i, 2}
\end{array}\right\}, \forall i \in V^{\text {Feeder }} \text {; (Equation 10) } \\
& \left.\begin{array}{l}
\left\|\left(2 v_{i, 1}, a_{i}-q_{i}\right)\right\|_{2} \leq a_{i}+q_{i} \\
\left\|\left(2, a_{i}-v_{i, 1}\right)\right\|_{2} \leq a_{i}+v_{i, 1}
\end{array}\right\}, \forall i \in V^{\text {Medium }} ; \\
& \left.\begin{array}{l}
\left\|\left(2 v_{i, 1}, a_{i}-1\right)\right\|_{2} \leq a_{i}+1 \\
\left\|\left(2 v_{i, 2}, v_{i, 2}-q_{i}\right)\right\|_{2} \leq v_{i, 1}+q_{i} \\
\left\|\left(2 v_{i, 3}, a_{i}-v_{i, 2}\right)\right\|_{2} \leq a_{i}+v_{i, 2} \\
\left\|\left(2 v_{i, 4}, v_{i, 1}-1\right)\right\|_{2} \leq v_{i, 1}+1 \\
\left\|\left(2, v_{i, 3}-v_{i, 4}\right)\right\|_{2} \leq v_{i, 3}+v_{i, 4}
\end{array}\right\}, \forall i \in V^{\text {Jumbo }} \\
& a_{i}, t_{i}, x_{i} \geq 0, \sigma_{i j}^{X} \in\{0,1\},
\end{aligned}
$$$$
\sigma_{i j}^{T} \in\{0,1\}, \forall i, j \text {; }
$$$$
q_{i}, v_{i, 1}, v_{i, 2}, v_{i, 3}, v_{i, 4} \geq 0, \forall i \text {. }
$$

\section{References}

Alizadeh, F; Goldfarb, D. 2003. Second-order cone programming, Mathematical Programming 95(1): 3-51. https://doi.org/10.1007/s10107-002-0339-5

Bierwirth, C.; Meisel, F. 2010. A survey of berth allocation and quay crane scheduling problems in container terminals, European Journal of Operational Research 202(3): 615-627. https://doi.org/10.1016/j.ejor.2009.05.031

Bierwirth, C.; Meisel, F. 2015. A follow-up survey of berth allocation and quay crane scheduling problems in container terminals, European Journal of Operational Research 244(3): 675-689. https://doi.org/10.1016/j.ejor.2014.12.030

Browning, L.; Façanha, C.; Papson, A.; Ang-Olson, J.; Hartley, S.; Carr, E. 2010. Representing Freight in Air Quality and Greenhouse Gas Models. National Cooperative Freight Research Program (NCFRP) Report 4. Transportation Research Board, Washington, DC, US. 169 p. https://doi.org/10.17226/14407 
Chang, D.; Jiang, Z.; Yan, W.; He, J. 2010. Integrating berth allocation and quay crane assignments, Transportation Research Part E: Logistics and Transportation Review 46(6): 975-990. https://doi.org/10.1016/j.tre.2010.05.008

Choi, B.-C.; Lee, K.; Leung, J. Y.-T.; Pinedo, M. L.; Briskorn, D. 2012. Container scheduling: complexity and algorithms, Production and Operations Management 21(1): 115-128. https://doi.org/10.1111/j.1937-5956.2011.01238.x

Corbett, J. J.; Fischbeck, P. 1997. Emissions from ships, Science 278(5339): 823-824.

https://doi.org/10.1126/science.278.5339.823

COSCO. 2009. COSCO Sustainable Development Report 2008. China Ocean Shipping Group (COSCO). 191 p. Available from Internet: http://en.coscoshipping.com/module/download/ downfile.jsp? filename $=370 \mathrm{bc} 81 \mathrm{c} 14 \mathrm{~d} 94 \mathrm{c} 29 \mathrm{~b} 240183 \mathrm{a} 2 \mathrm{cbf} 44$ 49.pdf

Dedes, E. K.; Hudson, D. A.; Turnock, S. R. 2012. Assessing the potential of hybrid energy technology to reduce exhaust emissions from global shipping, Energy Policy 40: 204-218. https://doi.org/10.1016/j.enpol.2011.09.046

Du, Y.; Chen, Q.; Quan, X.; Long, L.; Fung, R. Y. K. 2011. Berth allocation considering fuel consumption and vessel emissions, Transportation Research Part E: Logistics and Transportation Review 47(6): 1021-1037. https://doi.org/10.1016/j.tre.2011.05.011

Elwany, M. H.; Ali, I.; Abouelseoud, Y. 2013. A heuristics-based solution to the continuous berth allocation and crane assignment problem, Alexandria Engineering Journal 52(4): 671677. https://doi.org/10.1016/j.aej.2013.09.001

Eyring, V.; Isaksen, I. S. A.; Berntsen, T.; Collins, W. J.; Corbett, J. J.; Endresen, O.; Grainger, R. G.; Moldanova, J.; Schlager, H.; Stevenson, D. S. 2010. Transport impacts on atmosphere and climate: shipping, Atmospheric Environment 44(37): 47354771. https://doi.org/10.1016/j.atmosenv.2009.04.059

Fagerholt, K.; Gausel, N. T.; Rakke, J. G.; Psaraftis, H. N. 2015. Maritime routing and speed optimization with emission control areas, Transportation Research Part C 52: 57-73.

https://doi.org/10.1016/j.trc.2014.12.010

Fagerholt, K.; Laporte, G.; Norstad, I. 2010. Reducing fuel emissions by optimizing speed on shipping routes, Journal of the Operational Research Society 61(3): 523-529.

https://doi.org/10.1057/jors.2009.77

Fagerholt, K.; Psaraftis, H. N. 2015. On two speed optimization problems for ships that sail in and out of emission control areas, Transportation Research Part D: Transport and Environment 39: 56-64. https://doi.org/10.1016/j.trd.2015.06.005

Franc, P.; Sutto, L. 2014. Impact analysis on shipping lines and European ports of a cap-and-trade system on $\mathrm{CO}_{2}$ emissions in maritime transport, Maritime Policy \& Management: the Flagship Journal of International Shipping and Port Research 41(1): 61-78. https://doi.org/10.1080/03088839.2013.782440

Fransoo, J. C.; Lee, C.-Y. 2013. The critical role of ocean container transport in global supply chain performance, Production and Operations Management 22(2): 253-268.

https://doi.org/10.1111/j.1937-5956.2011.01310.x

Gibbs, D.; Rigot-Muller, P.; Mangan, J.; Lalwani, C. 2014. The role of sea ports in end-to-end maritime transport chain emissions, Energy Policy 64: 337-348.

https://doi.org/10.1016/j.enpol.2013.09.024

Golias, M. M.; Saharidis, G. K.; Boile, M.; Theofanis, S.; Ierapetritou, M. G. 2009. The berth allocation problem: optimizing vessel arrival time, Maritime Economics \& Logistics 11(4): 358-377. https://doi.org/10.1057/mel.2009.12
Guan, Y.; Cheung, R. K. 2004. The berth allocation problem: models and solution methods, OR Spectrum 26(1): 75-92. https://doi.org/10.1007/s00291-003-0140-8

Hendriks, M.; Laumanns, M.; Lefeber, E.; Udding, J. T. 2010. Robust cyclic berth planning of container vessels, OR Spectrum 32(3): 501-517. https://doi.org/10.1007/s00291-010-0198-Z

Hu, Q.-M.; Hu, Z.-H.; Du, Y. 2014. Berth and quay-crane allocation problem considering fuel consumption and emissions from vessels, Computers \& Industrial Engineering 70: 1-10. https://doi.org/10.1016/j.cie.2014.01.003

Kim, K. H.; Moon, K. C. 2003. Berth scheduling by simulated annealing, Transportation Research Part B: Methodological 37(6): 541-560. https://doi.org/10.1016/S0191-2615(02)00027-9

Kontovas, C.; Psaraftis, H. N. 2011. Reduction of emissions along the maritime intermodal container chain: operational models and policies, Maritime Policy \& Management: the Flagship Journal of International Shipping and Port Research 38(4): 451-469. https://doi.org/10.1080/03088839.2011.588262

Lim, A. 1998. The berth planning problem, Operations Research Letters 22(2-3): 105-110.

https://doi.org/10.1016/S0167-6377(98)00010-8

Lindstad, H.; Sandaas, I.; Strømman, A. H. 2015. Assessment of cost as a function of abatement options in maritime emission control areas, Transportation Research Part D: Transport and Environment 38: 41-48.

https://doi.org/10.1016/j.trd.2015.04.018

Masiol, M.; Harrison, R. M. 2014. Aircraft engine exhaust emissions and other airport-related contributions to ambient air pollution: a review, Atmospheric Environment 95: 409-455. https://doi.org/10.1016/j.atmosenv.2014.05.070

Mavrotas, G. 2009. Effective implementation of the $\varepsilon$-constraint method in multi-objective mathematical programming problems, Applied Mathematics and Computation 213(2): 455-465. https://doi.org/10.1016/j.amc.2009.03.037

Park, K. T.; Kim, K. H. 2002. Berth scheduling for container terminals by using a sub-gradient optimization technique, Journal of the Operational Research Society 53(9): 1054-1062. https://doi.org/10.1057/palgrave.jors.2601412

Park, Y.-M.; Kim, K. H. 2003. A scheduling method for berth and quay cranes, OR Spectrum 25(1): 1-23. https://doi.org/10.1007/s00291-002-0109-z

Raa, B.; Dullaert, W.; Schaeren, R. V. 2011. An enriched model for the integrated berth allocation and quay crane assignment problem, Expert Systems with Applications 38(11): 1413614147. https://doi.org/10.1016/j.eswa.2011.04.224

Rodriguez-Molins, M.; Ingolotti, L.; Barber, F.; Salido, M. A.; Sierra, M. R.; Puente, J. 2014. A genetic algorithm for robust berth allocation and quay crane assignment, Progress in Artificial Intelligence 2(4): 177-192. https://doi.org/10.1007/s13748-014-0056-3

Schrooten, L.; De Vlieger, I.; Panis, L. I.; Chiffi, C.; Pastori, E. 2009. Emissions of maritime transport: a European reference system, Science of the Total Environment 408(2): 318-323. https://doi.org/10.1016/j.scitotenv.2009.07.037

Seyedalizadeh Ganji, S. R.; Babazadeh, A.; Arabshahi, N. 2010. Analysis of the continuous berth allocation problem in container ports using a genetic algorithm, Journal of Marine Science and Technology 15(4): 408-416. https://doi.org/10.1007/s00773-010-0095-9

Song, D.-P.; Xu, J. 2012. An operational activity-based method to estimate $\mathrm{CO}_{2}$ emissions from container shipping considering empty container repositioning, Transportation Research Part D: Transport and Environment 17(1): 91-96. https://doi.org/10.1016/j.trd.2011.06.007 
Stahlbock, R.; Voß, S. 2008. Operations research at container terminals: a literature update, OR Spectrum 30(1): 1-52. https://doi.org/10.1007/s00291-007-0100-9

Starcrest Consulting Group. 2011. Port of Los Angles Inventory of Air Emissions - 2010. Technical Report ADP\#050520-525. Starcrest Consulting Group, LLC, Poulsbo, WA, US. 226 p. Available from Internet: https://kentico.portoflosangeles.org/ getmedia/26d2bb85-c08c-4776-afce-40677296e048/2010_ Air_Emissions_Inventory

Steenken, D.; Voß, S.; Stahlbock, R. 2004. Container terminal operation and operations research - a classification and literature review, OR Spectrum 26(1): 3-49. https://doi.org/10.1007/s00291-003-0157-z

Svindland, M. 2018. The environmental effects of emission control area regulations on short sea shipping in Northern Europe: the case of container feeder vessels, Transportation Research Part D: Transport and Environment 61: 423-430. https://doi.org/10.1016/j.trd.2016.11.008

Vis, I. F. A.; De Koster, R. 2003. Transshipment of containers at a container terminal: an overview, European Journal of Operational Research 147(1): 1-16. https://doi.org/10.1016/S0377-2217(02)00293-X

Wang, F.; Lim, A. 2007. A stochastic beam search for the berth allocation problem, Decision Support Systems 42(4): 21862196. https://doi.org/10.1016/j.dss.2006.06.008

Yang, C.; Wang, X.; Li, Z. 2012. An optimization approach for coupling problem of berth allocation and quay crane assignment in container terminal, Computers \& Industrial Engineering 63(1): 243-253. https://doi.org/10.1016/j.cie.2012.03.004

Yau, P. S.; Lee, S.-C.; Ho, K. F. 2012. Speed profiles for improvement of maritime emission estimation, Environmental Engineering Science 29(12): 1076-1084.

https://doi.org/10.1089/ees.2011.0399

Zeng, Q.; Hu, X.; Wang, W.; Fang, Y. 2011a. Disruption management model and its algorithms for berth allocation problem in container terminals, International Journal of Innovative Computing, Information and Control 7(5): 2763-2773.

Zeng, Q.; Yang, Z.; Hu, X. 2011b. Disruption recovery model for berth and quay crane scheduling in container terminals, Engineering Optimization 43(9): 967-983. https://doi.org/10.1080/0305215X.2010.528411

Zhen, L.; Chew, E. P.; Lee, L. H. 2011. An integrated model for berth template and yard template planning in transshipment hubs, Transportation Science 45(4): 483-504.

https://doi.org/10.1287/trsc.1100.0364 